\title{
Single top quark production as a window to physics beyond the standard model
}

\author{
Tim M. P. Tait* \\ Argonne National Laboratory, Argonne, Illinois 60439 \\ C.-P. Yuan ${ }^{\dagger}$ \\ Theory Division, CERN, CH-1211 Geneva, Switzerland
}

(Received 27 July 2000; revised manuscript received 20 September 2000; published 11 December 2000)

Production of single top quarks at a high energy hadron collider is studied as a means to identify physics beyond the standard model related to the electroweak symmetry breaking. The sensitivity of the $s$-channel $W^{*}$ mode, the $t$-channel $W$-gluon fusion mode, and the $t W^{-}$mode to various possible forms of new physics is assessed, and it is found that the three modes are sensitive to different forms of new physics, indicating that they provide complimentary information about the properties of the top quark. Polarization observables are also considered, and found to provide potentially useful information about the structure of the interactions of top quarks.

DOI: 10.1103/PhysRevD.63.014018

PACS number(s): 14.65.Ha, 12.39.Fe, 12.60.-i

\section{INTRODUCTION}

With the discovery of the top quark at the Fermilab Tevatron [1], the third generation of fermions in the standard model (SM) is complete. However, the question remains, is the top quark "just another quark?" or "is it something more?" The top quark is the only quark to have a mass on the same order as electroweak symmetry breaking (EWSB) scale, $v \sim 246 \mathrm{GeV}$, whereas all other observed fermions have masses that are a tiny fraction of this energy. This enormous mass may be a clue that the top quark plays a special role in EWSB. Following that line of thinking, many of the proposed extensions of the SM explain the large top quark mass by allowing the top quark to participate in new dynamics [2-6], which is connected to the physics providing the mass of the $W$ and $Z$ bosons. Thus, one of the primary motivations for the Tevatron run II and CERN Large Hadron Collider (LHC) ${ }^{1}$ is to accurately determine the top quark's properties, to see if any hint of nonstandard physics may be visible, and thus provide information about the mechanism of mass generation $[7,8]$.

Top quarks may be produced in pairs at a hadron collider via the strong interaction, through processes such as $q \bar{q}$ $\rightarrow t \bar{t}$ and $g g \rightarrow t \bar{t}$. Thus, the rate and kinematic distributions of top quarks produced in this way are a measure of the top quarks interactions with the gluons. The top quark decay proceeds via the weak interaction, and as we shall see does provide interesting information about the chiral structure of the $W-t-b$ interaction [7,9]. However, decays are experimentally relatively insensitive to the magnitude of the interaction by which they are mediated. For example, in the case of the

\footnotetext{
*Email address: tait@anl.gov

${ }^{\dagger}$ On leave from Department of Physics and Astronomy, Michigan State University, East Lansing, Michigan 48824. Email address: yuan@pa.msu.edu

${ }^{1}$ The Tevatron Run II is a proton-antiproton collider with $\sqrt{S}=2$ $\mathrm{TeV}$, and the LHC is a proton-proton collider with $\sqrt{S}=14 \mathrm{TeV}$.
}

top quark, there is one SM decay mode, $t \rightarrow b W^{+}$. If this vertex were somehow modified by new physics to have a different magnitude, it would affect the top quark's intrinsic width. However, at a hadron collider the width cannot be measured because the experimental resolutions are much larger than the width itself [10]. Similarly, while observing exotic top quark decays would certainly be interesting and would suggest what type of new vertices describe the observed decays, it would not determine the magnitude of these new interactions. Even a study of branching fractions compared to the SM decay mode may be misleading, because one must have already measured the $W-t-b$ interaction strength itself through some other means.

These drawbacks lead one to study weak production mechanisms of the top quark, which have cross sections directly proportional to the top quark's weak couplings. The $Z$ - $t$ - $t$ coupling will presumably contribute to $t \bar{t}$ production, though distinguishing this from the much larger QCD $t \bar{t}$ production is most likely hopeless at hadron colliders. [Nevertheless, it can be precisely measured at an electron linear collider (LC) [11].] A better process to measure this interaction is the production of $t \bar{t} Z$, where the $Z$ can be observed directly. The $W-t-b$ interaction will allow one to produce single top quarks at a hadron collider, and thus directly measure the properties of this interaction. A further consideration is that new physics characterized by an energy scale $\Lambda$ may be more apparent in higher energy processes, closer to $\Lambda$.
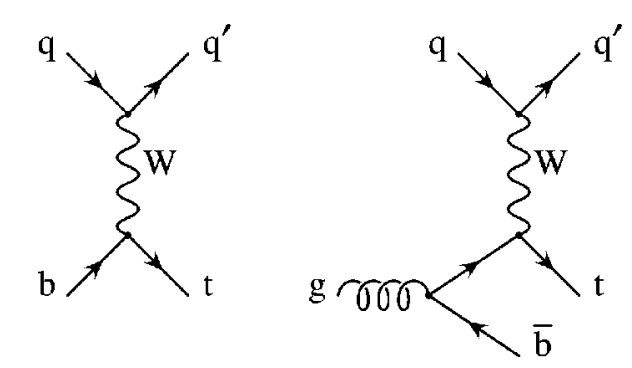

FIG. 1. Feynman diagrams for $t$-channel production of a single top quark in the SM. 


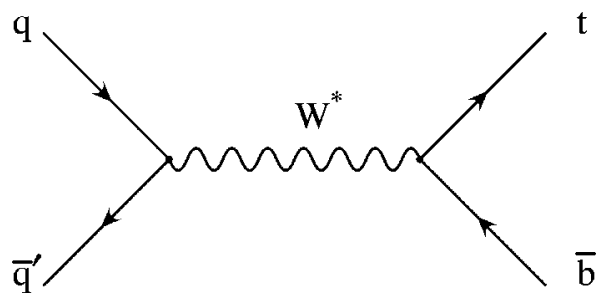

FIG. 2. Feynman diagram for $s$-channel production of a single top quark in the SM.

Thus, new physics contributions to single top quark production would scale as $(\sqrt{s} / \Lambda)^{n}$ (where $s$ is the average center of mass energy of the reaction and $n$ is a positive integer or zero) whereas top quark decay processes scale as $\left(m_{t} / \Lambda\right)^{n}$. At high energy colliders such as the Tevatron and LHC, $\sqrt{s}$ can be considerably larger than $m_{t}$, thus enhancing the relative importance of new physics in single top quark production.

Single top quark production proceeds through three distinct subprocesses at a hadron collider. The $t$-channel $W$-gluon fusion mode [12-14] involves the exchange of a spacelike $W$ boson between a light quark and a bottom $(b)$ quark inside the incident hadrons, resulting in a jet and a single top quark (cf. Fig. 1). Its rate ${ }^{2}$ is rather large at both the Tevatron $(2.4 \mathrm{pb})$ and the LHC (243 pb). The $s$-channel $W^{*}$ mode [16] involves production of an off-shell, time-like $W$ boson, which then decays into a top and a bottom quark (cf. Fig. 2). It has a relatively large rate at the Tevatron (0.86 $\mathrm{pb})$, but is comparatively small at the LHC (11 pb) because it is driven by initial state antiquark parton densities. Finally, the $t W^{-}$mode $[18,19]$ of single top quark production involves an initial state $b$ quark emitting a (close to) on-shell $W^{-}$boson, resulting in a $t W^{-}$final state (cf. Fig. 3). Because of the massive particles in the final state, this mode has an extremely small rate at the Tevatron $(0.088 \mathrm{pb})$, but is considerable at the LHC (51 pb) where more partonic energy is available. Each mode has rather distinct event kinematics, and thus are potentially observable separately from each other $[13,14,19]$.

As we consider various new physics effects in single top quark production below, it will be important to keep in mind how accurately these rates will be measured at the Tevatron Run II and LHC. At the Tevatron, the combined statistical and theoretical uncertainties for the $s$ - and $t$-channel processes will be on the order of $10-20 \%$ [17], and the $t W^{-}$rate will most likely be too small to measure. ${ }^{3}$ At the LHC, the statistical uncertainties will be close to zero, and the theoretical uncertainties on the order of $5-10 \%$ for the

\footnotetext{
${ }^{2}$ In quoting cross sections, we use a top quark mass of $175 \mathrm{GeV}$ and CTEQ4M (for $s$ - and $t$-channel processes) and CTEQ4L (for the $t W^{-}$mode) parton densities [15] with scales chosen as explained in [7], where other choices of parton densities, scale, and top quark mass are also examined. The rates of $t$ and $\bar{t}$ are summed unless otherwise noted.

${ }^{3}$ These estimates do not include systematic uncertainties, which are beyond the scope of this work.
}
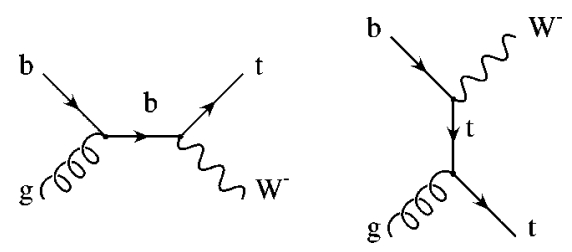

FIG. 3. Feynman diagrams for $t W^{-}$production in the SM.

$s$ - and $t$-channel processes [17], and on the order of $20 \%$ for the $t W^{-}$process [19]. However, systematic uncertainties (including the efficiency for separating the signal from the backgrounds) are likely to dominate these LHC estimates.

As we will see below, the three modes of single top quark production are sensitive to quite different manifestations of physics beyond the standard model. Thus, taken together, they are a comprehensive probe of the top quark's interactions. In this article, we analyze several possible signals of new physics that could manifest themselves in single top quark production. These signals can be classified as to whether they involve the effects of a new particle (either fundamental or composite) that couple to the top quark, or the effect of a modification of the SM coupling between the top and other known particles. These two classifications can be seen to overlap in the limit in which the additional particles are heavy and decouple from the low energy description. In this case the extra particles are best seen through their effects on the couplings of the known particles. One particular aspect of both of these sets of new physics is the possibility of $C P$ violation in top observables. We will not address this issue in detail; the interested reader is referred to the recent review [20] on this subject.

This article is organized as follows: in Sec. II, we investigate what sort of particles beyond those in the SM could contribute to single top quark production at a hadron collider, and what effects could be seen. In Sec. III, we study the effects of nonstandard top quark interactions. In Sec. IV, we examine how one may use polarization observables to further learn about nonstandard physics in the top sector. In Sec. V we summarize the picture which emerges as the way in which the properties of a top quark may be systematically extracted from the available observables.

\section{ADDITIONAL NONSTANDARD PARTICLES}

Many theories of physics beyond the SM predict the existence of particles beyond those required by the SM itself. Examples include both the fundamental superpartners in a theory with supersymmetry (SUSY) [21], and the composite Higgs bosons found in top-condensation and top-color models [2-6]. In order for some kind of additional particle to contribute to single top quark production at tree level ${ }^{4}$ at a hadron collider, the new particle must somehow couple the top quark to one of the lighter SM particles. Thus, the new

\footnotetext{
${ }^{4} \mathrm{New}$ particles may also contribute to single top quark production through loops [22], though the effects are generally small enough that they are difficult to observe at a hadron collider.
} 
particle may be either a boson (such as a $W^{\prime}$ vector boson that couples to top and bottom) or a fermion (such as a $b^{\prime}$ quark that couples to the $W$ boson and top).

Additional fermionic particles can couple the top and either one of the gauge bosons or the Higgs boson. In order to respect the color symmetry, this requires that the extra fermion occurs in a color triplet, and thus it is sensible to think of it as some type of quark. In order to be invariant under the electromagnetic symmetry, this new "quark" should have either electric charge $(Q)+2 / 3$ or $-1 / 3$ so that one may construct gauge invariant interactions between the extra quark, the top quark, and the known bosons. Generally, we can refer to a $Q=+2 / 3$ extra quark as a $t^{\prime}$ and a $Q=-1 / 3$ extra quark as a $b^{\prime}$, though this does not necessarily imply that the extra quarks are in the same representation under $\mathrm{SU}(2)_{L} \times \mathrm{U}(1)_{Y}$ as the SM top and bottom. For example, in models where the top mass is generated by invoking a seesaw mechanism, there are generally either SU(2) singlet [3] or doublet [6] quarks present in the theory. Additional fermions are not generally expected to be a large source of new contributions to single top quark production, because of strong constraints from other low energy observables [23]. On the other hand we will see that there are models with additional fermions to which single top production is a sensitive probe.

Extra bosons can contribute to single top quark production either by coupling top to the down-type quarks, in which case the boson must have electric charge $Q= \pm 1$ in order to maintain the electromagnetic symmetry, or by coupling top to the charm or up quarks, in which case the boson should be electrically neutral. There is also the possibility of a boson carrying a combination of color and electric charge that allows it to couple the top quark to one of the lepton fields (this boson would then carry both lepton and baryon quantum numbers and thus may be labeled a "leptoquark"). Theoretically well motivated examples of leptoquarks are the gauge bosons corresponding to the generators of a grand unified theory (GUT) linking the $\mathrm{SU}(2)$ and $\mathrm{SU}(3)$ sectors of the SM. This GUT picture has the leptoquark as part of the gauge interactions, so the question as to whether or not top observables are an interesting means to study leptoquarks becomes a question as to whether or not the leptoquark has some reason to prefer to couple to the top quark. These bosons would be expected to have GUT scale masses which in traditional GUT theories is far too large to be interesting from the point of view of colliders envisioned in the near future. $^{5}$

Another interesting picture of leptoquarks is one in which the SM quarks and leptons are bound states of some more fundamental set of particles (preons). In that case the question as to whether or not the top quark is a good place to look for evidence of the preons depends on how the model arranges the various types of preons to build quarks and lep-

\footnotetext{
${ }^{5}$ However, a GUT theory in five space-time dimensions could unify at the TeV scale [24] and would be accessible to the current generation of hadron colliders through a number of observables.
}

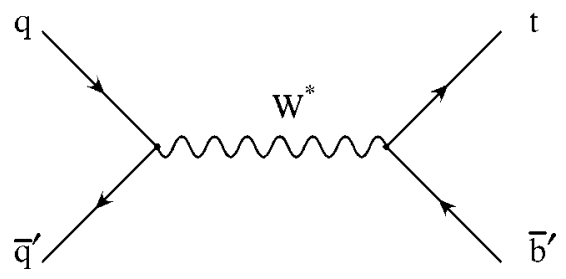

FIG. 4. Feynman diagram for $s$-channel production of a single top quark and a $b^{\prime}: q \bar{q}^{\prime} \rightarrow t \bar{b}^{\prime}$.

tons. However, at a hadron collider the possible light parton initial states available are not suitable for production of a single leptoquark, and thus are not particularly interesting in the context of single top quark production. ${ }^{6}$ For this reason, we will not focus on leptoquarks in the discussion below.

\section{A. Extra quarks}

A simple extension of the SM is to allow for an extra set of quarks. Such objects exist in a wide variety of extensions to the SM. Examples of such theories include the top seesaw versions of the top-color [3] and top-flavor [6] models, which rely on additional fermions to participate in a seesaw mechanism to generate the top quark mass; SUSY theories with gauge mediated SUSY breaking [26] that must be communicated from a hidden sector in which SUSY is broken to the visible sector through the interactions of a set of fields with SM gauge quantum numbers; and even models with a fourth generation of fermions.

Direct search limits for QCD production of extra quarks (at the Tevatron, for example) require that they be quite massive $\left(m_{q^{\prime}} \geqslant 46-128 \mathrm{GeV}\right.$ at the $95 \%$ C.L., depending on the decay mode [23]), and thus they cannot significantly affect the single top quark production rate. They are best observed either through their mixings with the third family (and thus their effect on the top quark couplings), or through direct production.

As a particular example, a fourth generation of quarks could mix with the third generation through a generalized Cabibbo-Kobayashi-Maskawa (CKM) matrix, and this could allow $V_{t b}$ to deviate considerably from unity. In this case, all three modes of single top quark production would be expected to have considerably lower cross sections than the SM predicts. This already shows how the separate modes of single top quark production can be used to learn about physics beyond the SM. Other types of new physics could scale the three rates independently. Thus, if all three modes are measured to have cross sections that are the same fraction of the SM rates, it is an indication that the new physics modifies the top quark's coupling to the bottom quark and $W$ (and not another pair of light particles), and further that the modification is the same regardless of the momentum flowing through

\footnotetext{
${ }^{6}$ It is interesting to note that a leptoquark $(L)$ with $Q=+2 / 3$ could play an important role in top quark decays through a process such as $t \rightarrow \nu L \rightarrow \nu b l^{+}$[25]. This leads to a final state that is identical to a SM top decay, but with a very distinct kinematic structure.
} 
the vertex (as is the case with the $W-t-b$ interaction in the SM).

In fact, it is instructive to examine the experimental constraints on the CKM matrix when one does not impose three family unitarity. The $90 \%$ C.L. direct constraints become [23]

$$
V=\left(\begin{array}{cccc}
0.9722-0.9748 & 0.216-0.223 & 0.002-0.005 & \ldots \\
0.199-0.233 & 0.784-0.976 & 0.037-0.043 & \ldots \\
0-0.09 & 0.0-0.55 & 0.06-0.9993 & \ldots \\
\ldots & \ldots & \ldots & \ldots
\end{array}\right)
$$

with two striking differences from the 3 generation matrix; $V_{t b}$ may be significantly smaller than unity (as mentioned above) but also that $V_{t s}$ may be as large as 0.55 . (For comparison, the corresponding limit on $V_{t s}$ with 3 generations is $\left.0.035 \leqslant\left|V_{t s}\right| \leqslant 0.043[23]\right) .\left|V_{t s}\right|=0.043$ has a negligible effect on the $t$-channel rate of single top quark production, contributing much less than $1 \%$ of the total rate at either Tevatron or LHC. However, if $\left|V_{t s}\right|=0.55$ and $\left|V_{t b}\right|$ $=0.835$ (which saturates the unitarity requirement for any number of families ${ }^{7}$ ), the NLO $t$-channel rate will rise to about $4.07 \mathrm{pb}$ at the Tevatron Run II and $334 \mathrm{pb}$ at the LHC, thanks to the much larger $s$ quark parton density compared to the $b$ quark. These are huge deviations from the SM rates, and would be a clear indication of new physics. Under these conditions, the $s$-channel rate, which would fall to $\left|V_{t b}\right|^{2}$ $\sim 0.7$ of its SM rate. Similarly to the $t$-channel mode, the $t W^{-}$rate will be enhanced to $0.19 \mathrm{pb}$ at the Tevatron and $78.1 \mathrm{pb}$ at the LHC under these conditions.

In addition to mixing effects, one could also hope to observe direct production of one of the fourth generation quarks, through reactions such as $q \bar{q}^{\prime} \rightarrow t \bar{b}^{\prime}$, shown in Fig. 4. This process would provide further information on the family structure of the 4 generation model $\left(V_{t b^{\prime}}\right)$ not readily available from the QCD production of $b^{\prime} \bar{b}^{\prime}$. The production rates will depend on the magnitude of the $W-t-b^{\prime}$ coupling $\left(\left|V_{t b^{\prime}}\right|^{2}\right.$ in the model with a fourth family) and the mass of the $b^{\prime}$. In Fig. 5 we present the next leading order (NLO) rate for $t \bar{b}^{\prime}$ production (as well as $\bar{t} b^{\prime}$ production) without any decay BR's. Since the $\left|V_{t b^{\prime}}\right|^{2}$ dependence may be factored out, these rates assume $V_{t b^{\prime}}=1$. The collider signatures resulting from such a process depend on the decay modes available to the $b^{\prime}$. If $m_{b^{\prime}}>m_{t}+m_{W}$, it is likely to decay into a top quark and a $W^{-}$, and the events will have a $t \bar{t}$ pair with an additional $W^{ \pm}$boson. If this decay mode is not open, loop induced decays such as $b^{\prime} \rightarrow b \gamma$ may become important, resulting in a signature $t \bar{b}$ plus a hard photon whose invariant mass with the $b$ quark will reconstruct the mass of the $b^{\prime}$.

\footnotetext{
${ }^{7}$ This choice of $V_{t b}$ and $V_{t s}$ is consistent with the CDF measurement $\quad \Gamma\left(t \rightarrow W^{+} b\right) /\left[\Gamma\left(t \rightarrow W^{+} b\right)+\Gamma\left(t \rightarrow W^{+} s\right)+\Gamma\left(t \rightarrow W^{+} d\right)\right]$ $=0.87_{-0.30-0.11}^{+0.13+0.13}[27]$.
}

\section{B. Extra gauge bosons}

Another simple extension of the SM is to postulate the existence of a larger gauge group which somehow reduces to the SM gauge group at low energies. Such theories naturally have additional gauge bosons, some of which may prefer to couple to the top (or even the entire third family). Examples of such theories include the top-color [3] and top-flavor [4-6] models, and gauged-flavor symmetry models [28] which give special dynamics to the third family in order to explain the large top mass. As a specific example, we will consider the top-flavor model with an extra $\mathrm{SU}(2)_{h}$ gauge symmetry that generates a top quark mass through a seesaw effect [6].

This model has an overall gauge symmetry of $\mathrm{SU}(3)_{C}$ $\times \mathrm{SU}(2)_{h} \times \mathrm{SU}(2)_{l} \times \mathrm{U}(1)_{Y}$, and thus there are three additional weak bosons ( $W^{\prime \pm}$ and $Z^{\prime}$ ). The first and second generation fermions and third family leptons transform under $\mathrm{SU}(2)_{l}$, while the third generation quarks transform under $\mathrm{SU}(2)_{h}$. As was alluded to before, in order to cancel the anomaly and provide a seesaw mechanism to generate the

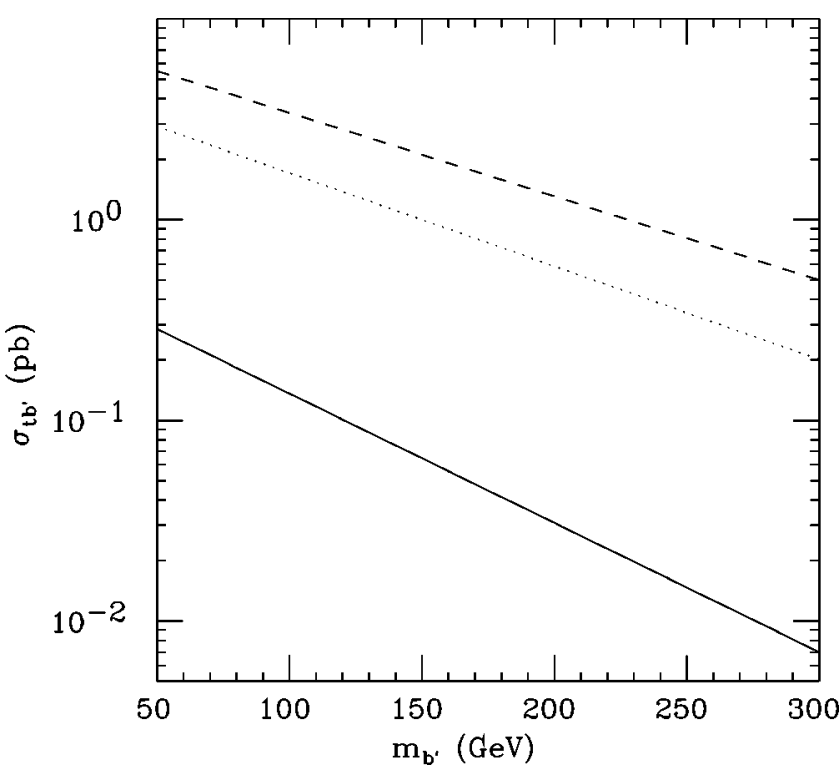

FIG. 5. The NLO rates (in pb) for the process $q \bar{q}^{\prime} \rightarrow W^{*}$ $\rightarrow t \bar{b}^{\prime}$ for various $b^{\prime}$ masses at the Tevatron (solid curve) and CERN LHC (dashed curve), assuming $V_{t b^{\prime}}=1$. At the Tevatron, the rates of $q \bar{q}^{\prime} \rightarrow W^{*} \rightarrow \bar{t} b^{\prime}$ is equal to the $t \bar{b}^{\prime}$ rate. The $\bar{t} b^{\prime}$ rate at the CERN LHC is shown as the dotted curve. 


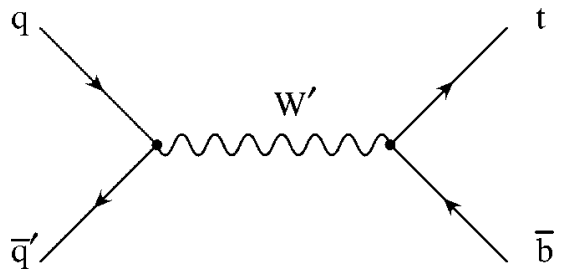

FIG. 6. Feynman diagrams illustrating how a $W^{\prime}$ boson can contribute to single top quark production through $q \bar{q}^{\prime} \rightarrow W^{\prime} \rightarrow t \bar{b}$.

top quark mass, an additional doublet of heavy quarks whose left-handed components transform under $\mathrm{SU}(2)_{l}$ and righthanded components transform under $\mathrm{SU}(2)_{h}$ is also present.

A set of scalar fields transforming under both $\mathrm{SU}(2)_{l}$ and $\mathrm{SU}(2)_{h}$ acquire a vacuum expectation valve (VEV), $u$, and break the symmetry to $\mathrm{SU}(2)_{l+h} \times \mathrm{U}(1)_{Y}$. From here the usual electroweak symmetry breaking can be accomplished by introducing a scalar doublet which acquires a $\operatorname{VEV~} v$, further breaking the gauge symmetry to $\mathrm{U}(1)_{E M}$. We write the covariant derivatives for the fermions as

$$
D^{\mu}=\partial^{\mu}+i g_{l} T_{l}^{a} W_{l}^{a \mu}+i g_{h} T_{h}^{a} W_{h}^{a \mu}+i g_{1} \frac{Y}{2} B^{\mu},
$$

where $T_{l(h)}^{a}$ are the generators for SU(2) $)_{l(h)}, Y$ is the hypercharge generator, and $W_{l(h)}^{a \mu}$ and $B^{\mu}$ are the gauge bosons for the $\mathrm{SU}(2)_{l(h)}$ and $\mathrm{U}(1)_{Y}$ symmetries. The gauge couplings may be written

$$
g_{l}=\frac{e}{\sin \theta_{W} \cos \phi}, \quad g_{h}=\frac{e}{\sin \theta_{W} \sin \phi}, \quad g_{1}=\frac{e}{\cos \theta_{W}},
$$

where $\phi$ is a new parameter in the theory. Thus this theory is determined by two additional quantities $x=u / v$, the ratio of the two VEV's, and $\sin ^{2} \phi$, which characterizes the mixing between the heavy and light $\mathrm{SU}(2)$ gauge couplings.

At leading order, the heavy bosons are degenerate in mass:

$$
M_{Z^{\prime}, W^{\prime}}^{2}=M_{0}^{2}\left(\frac{x}{\sin ^{2} \phi \cos ^{2} \phi}+\frac{\sin ^{2} \phi}{\cos ^{2} \phi}\right),
$$

where $M_{0}^{2}=e^{2} v^{2} / 4 \sin ^{2} \theta_{W} \cos ^{2} \theta_{W}$. We can thus parametrize the model by the heavy boson mass, $M_{Z^{\prime}}$, and the mixing parameter, ${ }^{8} \sin ^{2} \phi$. Low energy data requires that the mass of these heavy bosons, $M_{Z^{\prime}}$, be greater than about $900 \mathrm{GeV}$ $[5]$.

The additional $W^{\prime}$ boson can contribute to the $s$-channel mode of single top quark production through virtual exchange of a $W^{\prime}$ as shown in Fig. 6 [29]. In particular, if enough energy is available, the $W^{\prime}$ may be produced close to on-shell, and a resonant enhancement of the signal may re-

\footnotetext{
${ }^{8}$ As shown in [4], for $\sin ^{2} \phi \leqslant 0.04$, the third family fermion coupling to the heavy gauge bosons can become nonperturbative. Thus we restrict ourselves to considering $0.95 \geqslant \sin ^{2} \phi \geqslant 0.05$.
}

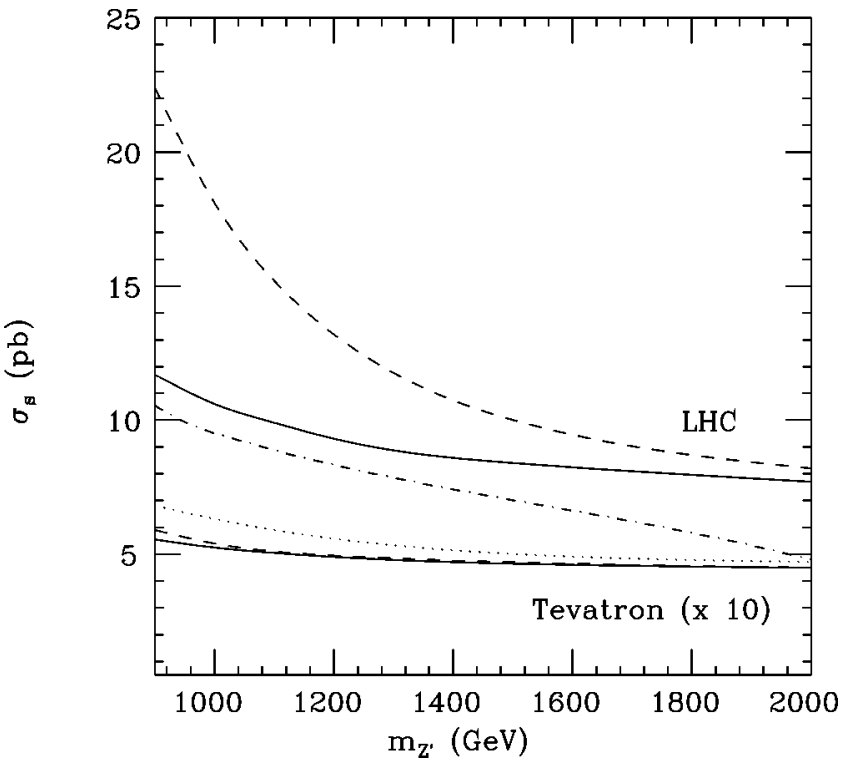

FIG. 7. The NLO rate of $q \bar{q}^{\prime} \rightarrow W, W^{\prime} \rightarrow t \bar{b}\left(\sigma_{S}\right)$ in $\mathrm{pb}$ at the Tevatron (lower curves) and CERN LHC (upper curves), for the top-quark-flavor model with $\sin ^{2} \phi=0.05$ (solid curves) and $\sin ^{2} \phi$ $=0.25$ (dashed curves), as a function of $M_{Z^{\prime}}=M_{W^{\prime}}$. The Tevatron cross sections are multiplied by a factor of 10. At the Tevatron, the $\bar{t}$ production rate is equal to the $t$ rate. At the CERN LHC the $\bar{t}$ rates are shown for $\sin ^{2} \phi=0.05$ (dotted curve) and $\sin ^{2} \phi=0.25$ (dot-dashed curve).

sult. Since the additional diagrams involve a virtual $W^{\prime}$, they will interfere with the SM $W$-exchange diagrams, and thus the net rate of single top quark production can be increased or decreased as a result, though the particular model under study always results in an increased $s$-channel single top quark rate. ${ }^{9}$ In Fig. 7 the resulting NLO $s$-channel rate for $q \bar{q}^{\prime} \rightarrow W, W^{\prime} \rightarrow t \bar{b}$ at Tevatron and LHC is shown, as a function of the $W^{\prime}$ mass, for a few values of $\sin ^{2} \phi$. The rate for $\bar{t}$ production through the same process is shown as well. While the final state particles for this case are the same as the SM $s$-channel mode, the distribution of the invariant mass of the $t \bar{b}$ system could show a Breit-Wigner resonance effect around $M_{W^{\prime}}$, which serves to identify this type of new physics. However, if the mass of the $W^{\prime}$ is large compared to the collider energy, and its width broad, the resonance shape can be washed out even at the parton level. Jet energy smearing from detector resolution effects will further degrade the resonance and could make it difficult to identify.

A $t$-channel exchange of the $W^{\prime}$ is also possible, but in that case a negligible effect is expected because the boson must have a spacelike momentum, and thus the additional contributions are suppressed by $1 / M_{W^{\prime}}^{2}$, and are not likely to be visible. This argument applies quite generally to any

\footnotetext{
${ }^{9}$ One interesting example of a model with a $W^{\prime}$ that interferes nontrivially with the SM $W$ exchange in single top quark production is provided by embedding the gauge fields in extra dimensions $[24,30]$.
} 


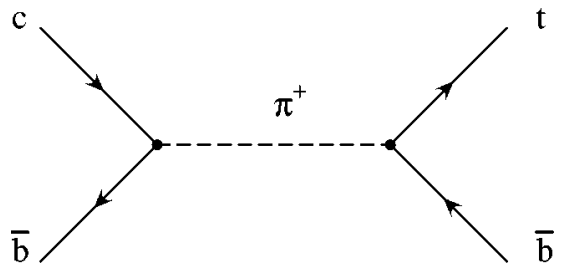

FIG. 8. Feynman diagram illustrating how a charged top-quark pion can contribute to single top quark production through $c \bar{b}$ $\rightarrow \pi^{+} \rightarrow t \bar{b}$.

heavy particle's effect on single top quark production. The $s$-channel rate is quite sensitive to a heavy particle because of the possibility of resonant production, whereas the $t$-channel rate is insensitive because the spacelike exchange is suppressed by the heavy particle mass.

Clearly, the existence of a $W^{\prime}$ will not influence the rate of $t W^{-}$production, but it could allow for exotic production modes such as $b g \rightarrow t W^{\prime}$. If the $W^{\prime}$ has a strong coupling with the third family, then one would expect that its dominant decay should be into $b \bar{t}$, and thus a final state of $t \bar{t} b$ would result with the $t \bar{b}$ invariant mass reconstructing the $W^{\prime}$ mass. Current limits on the $W^{\prime}$ mass in the top-flavor model make this mode nonviable at the Tevatron and unpromising at the LHC, with a cross section of $1.14 \mathrm{pb}$ for $M_{W^{\prime}}=900 \mathrm{GeV}$ and $\sin ^{2} \phi=0.05$ including the large QCD logarithmic corrections described in [19]. However, an observation of this signal would be a clear indication of the nature of the new physics.

\section{Extra scalar bosons}

Scalar particles appear in many theories, usually associated with the spontaneous breaking of a symmetry in a Lorentz invariant fashion. In the SM and the minimal supersymmetric extension, fundamental scalar fields of both neutral and charged character are present in the theory, and some are expected to have a strong coupling with the top quark because of the role they play in generating fermion masses. In dynamical models such as the top-condensate and top-color assisted technicolor models, scalar particles exist as bound states of top and bottom quarks [2-6]. These composite scalars also have a strong coupling to the top quark because of their role in the generation of the top quark mass. Another way to look at this is that the top quark mass is large because the strong forces needed to bind top quarks into Higg bosons result in a strong Higgs coupling to the top quark. This illustrates the fact that the large top mass naturally makes it a likely place to look for physics associated with the EWSB.

An example is provided by the charged composite toppions $\left(\pi^{ \pm}\right)$of the top-color model, which can be produced in the $s$-channel through $c \bar{b}$ fusion [31], $c \bar{b} \rightarrow \pi^{+} \rightarrow t \bar{b}$. The leading order Feynman diagram is shown in Fig. 8. In this case the strong $\pi^{+}-c-\bar{b}$ coupling comes from mixing between the right-handed $t$ and $c$ quarks. The CKM matrix is the product of the left-handed rotation matrices for the upand down-type quarks, so it does not constrain a possibly large right-handed $t$ and $c$ mixing. The fact that this interac-

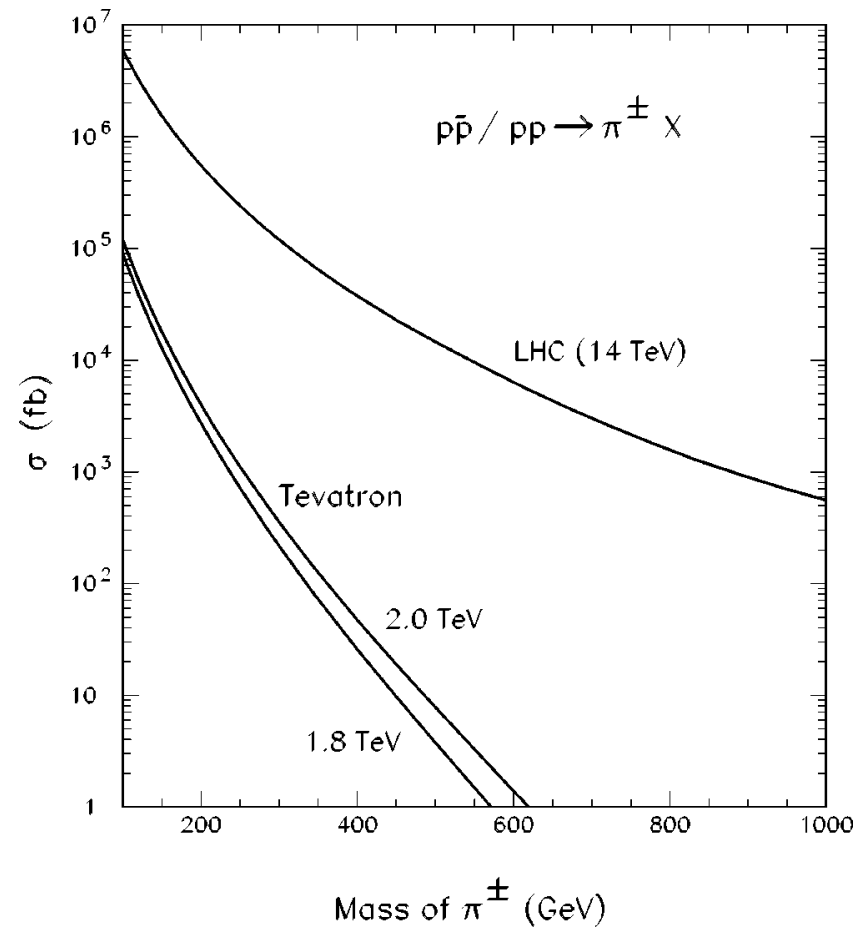

FIG. 9. The NLO rate of single top quark production through the reaction $c \bar{b} \rightarrow \pi^{+} \rightarrow t \bar{b}$ as a function of $M_{\pi^{ \pm}}$, assuming a $t_{R^{-}} c_{R}$ mixing of $20 \%$. These rates include $t$ and $\bar{t}$ production, which are equal for both Tevatron and CERN LHC.

tion has a right-handed nature will prove interesting when we study top quark polarization below.

Like the $W^{\prime}$, the $\pi^{ \pm}$contributes to the $s$-channel topology of single top quark production and can allow large resonant contributions. However, unlike the $W^{\prime}$, the $\pi^{+}$does not interfere with the SM amplitudes $\left(q^{\prime} \bar{q} \rightarrow t \bar{b}\right)$, because the SM contribution arises dominantly from (left-handed) light quarks. In Fig. 9, we present the NLO single top quark rate from the top-pion process [32], for a variety of $\pi^{ \pm}$masses with the $t_{R}-c_{R}$ mixing set equal to $20 \%$. The two other modes of single top quark production are once again relatively insensitive to the $\pi^{ \pm}$. The $t$-channel process is insensitive because its contribution is suppressed by $1 / M_{\pi^{ \pm}}^{2}$ and the fact that the $\pi^{ \pm}$does not couple to light quarks. The $t W^{-}$mode is insensitive because presumably the $\pi^{ \pm}$is generally distinguishable from a $W^{ \pm}$boson. For example, $g b$ $\rightarrow \pi^{-} t \rightarrow \bar{t} b t$ will not be mistaken for $t W^{-}$production

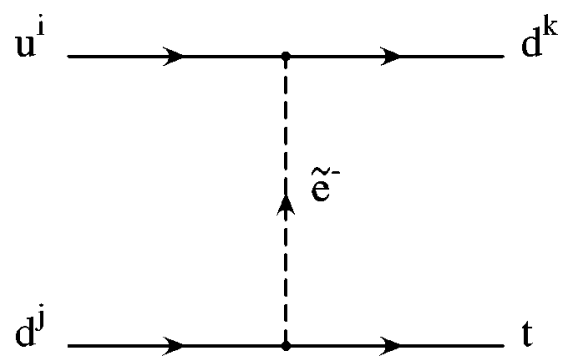

FIG. 10. Illustrative Feynman diagram for the $R$-parity violating production of a single top quark through the reaction $u^{i} d^{j} \rightarrow t d^{k}$. 


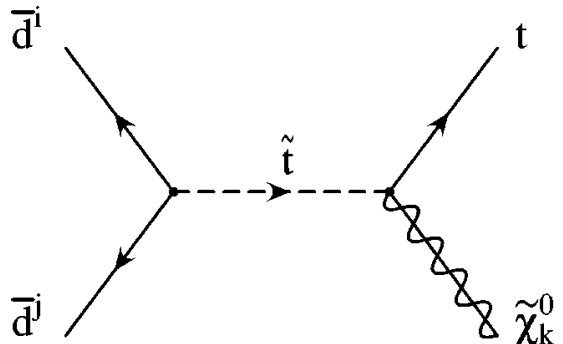

FIG. 11. Illustrative Feynman diagram for the $R$-parity violating production of a single top quark through the reaction $\bar{d}^{i} \bar{d}^{j} \rightarrow \tilde{t}$ $\rightarrow t \tilde{\chi}_{k}^{0}$.

when $\pi^{-}$predominantly decays into $\bar{t}$ and $b$, as in the topcolor model.

Single top quarks can also be produced by neutral toppions (produced for example from $g g \rightarrow \pi^{0}$ decaying into $t \bar{c})$. Again, one expects to see a large effect in the $s$-channel single top quark mode, and no effect in the $t$-channel and $t W^{-}$modes, because of the large $\pi^{0}$ mass and small coupling to light quarks [33].

Different types of scalar particles that couple the top and bottom quarks can be analyzed in a similar fashion. The $s$-channel mode allows for resonant production, which can show a large effect, whereas the $t$-channel mode is suppressed by the spacelike momentum (and large mass) of the exchanged massive particle. The $t W^{-}$mode is insensitive because in that case the $W$ is actually observed in the final state. One example of this kind is the technipions in a technicolor model which can contribute to single top quark production in this way [34]. Another example is provided by SUSY models with broken $R$-parity, in which the scalar partners of the leptons (sleptons) can couple with the top and bottom quarks, and will contribute to single top quark production [35], or through reactions such as $\bar{d} \bar{s} \rightarrow \tilde{t} \rightarrow t \tilde{\chi}_{i}^{0}[36]$. (See Figs. 10 and 11 for their representative Feynman diagrams.)

As a final note, there is the interesting process in which a neutral scalar (like the Higgs boson of the SM) is produced in association with a single top quark [37]. Feynman diagrams are shown in Fig. 12. This process is of interest because while the magnitude of the $h-W-W$ and $h-t-t$ couplings can be measured independently by studying $q \bar{q}^{\prime} \rightarrow W^{*}$ $\rightarrow W h$ and $q \bar{q}(g g) \rightarrow h t \bar{t}$ (or the equivalent processes at a high energy lepton collider), the relative phase between the

b
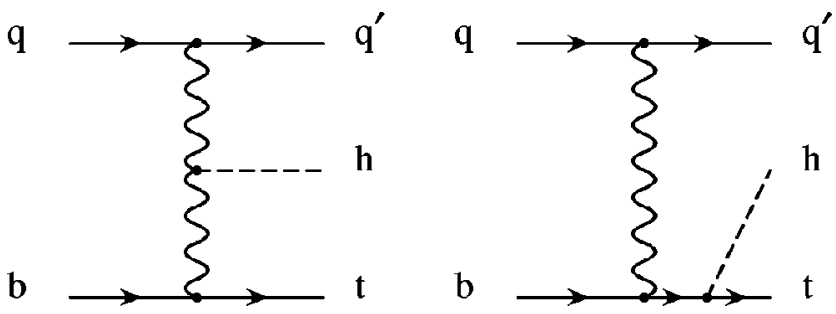

FIG. 12. Feynman diagrams for associated production of a neutral scalar and single top quark: $q b \rightarrow q^{\prime} t h$.

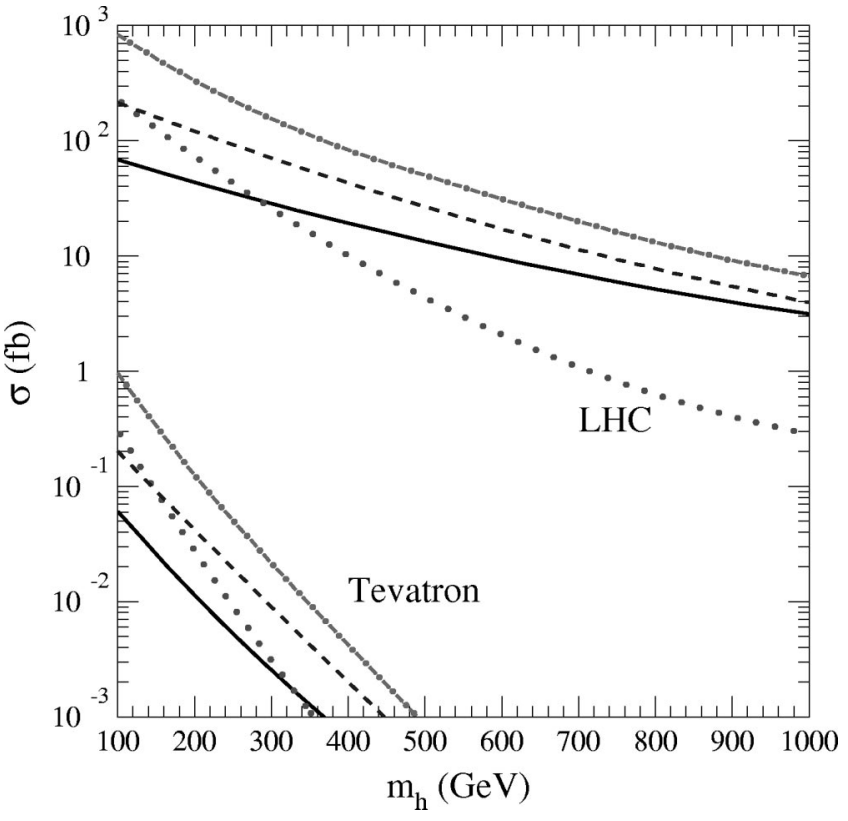

FIG. 13. The cross section for production of a Higgs boson in association with a single top quark, as a function of the Higgs boson mass at the Tevatron Run II (lower family of curves) and the CERN LHC (upper family of curves). The cross sections are shown for models in which the Higgs couplings are completely SM-like (solid curves), the coupling to the $W^{ \pm}$boson is zero and the coupling to a top quark is SM-like (dotted curves), the coupling to a top quark is zero and the coupling to $W^{ \pm}$is SM-like (dashed curves), and the coupling to $W^{ \pm}$is SM-like and the coupling to a top quark is -1 times the SM coupling (dash-dotted curves).

couplings can be found from the process $q b \rightarrow q^{\prime} t h$, as that phase information is contained in the interference between the two diagrams shown in Fig. 12. This process is extremely small compared to the other two mentioned [with a SM cross section of $4.4 \times 10^{-2} \mathrm{fb}$ at the Tevatron and $0.06 \mathrm{pb}$ at the LHC for a Higgs boson of mass $\left(m_{h}\right) 110 \mathrm{GeV}$, and including both $t$ and $\bar{t}$ production], and thus it is not promising a discovery mode. The small SM rate results from the large destructive interference between the two diagrams. Typically, it yields a reduction in the rate as compared to the contribution from each individual diagram by about an order of magnitude. In Fig. 13 we show the contributions from each Feynman diagram containing either $h-t-t$ or $h-W-W$ vertex to the total production rate of $q^{\prime} h t$ as a function of $m_{h}$ at Run II of the Tevatron and the LHC. Though the rates at the Tevatron are very small in the SM, with the enhanced coupling of $h-t-\bar{t}$ predicted by some models of new physics, this process could conceivably be observed there. The strong cancellation predicted by the SM indicates that this process is very sensitive to any physics that modifies the relative phase (and size) between the $h-t-t$ and $h-W-W$ couplings from the SM relation. Thus, it contains important information not available in the $W h$ and $h t \bar{t}$ processes, and should be carefully tested experimentally.

\section{MODIFIED TOP QUARK INTERACTIONS}

Another interesting set of properties of the top that can be studied in single top quark production are the top quark cou- 
plings to light particles. The electroweak chiral Lagrangian [38] (EWCL) provides a powerful way to study such effects model independently. Following the EWCL approach, we write an effective Lagrangian to describe low energy physics as

$$
\mathcal{L}_{\text {eff }}=\mathcal{L}_{S M}+\mathcal{L}_{4}+\mathcal{L}_{5}+\cdots,
$$

where $\mathcal{L}_{S M}$ refers to the usual SM Lagrangian, and $\mathcal{L}_{4}$ and $\mathcal{L}_{5}$ are the Lagrangians containing deviations from the SM in terms of operators of mass dimension 4 and 5, respectively. The spirit of the EWCL approach is that higher order operators will generally be suppressed by higher powers of $\Lambda$, the scale at which the effective theory breaks down. Thus, for low energy processes occurring at energies below $\Lambda$ the lowest dimension anomalous operator is expected to provide the largest effect [39]. We choose the effective Lagrangian to realize the weak symmetry nonlinearly, as this is the most general possibility [40]. This is most appropriate for a strongly interacting underlying theory, which may not contain a Higgs doublet in any real sense. If the underlying theory is weakly coupled, it is most likely more appropriate to describe the new physics effects in terms of a theory with the weak symmetry realized linearly, in which case all nonstandard effects will be suppressed by at least one power of $\Lambda$.

Terms which have the potential to modify single top quark production include mass dimension 4 operators [41],

$$
\begin{aligned}
\mathcal{L}_{4}= & \frac{e}{\sqrt{2} \sin \theta_{W}} W^{+}{ }_{\mu}\left(\kappa_{W t b}^{L} e^{i \phi_{W t b}^{L} \bar{b}} \gamma^{\mu} P_{L} t\right. \\
& \left.+\kappa_{W t b}^{R} e^{i \phi_{W t b}^{R} \bar{b}} \gamma^{\mu} P_{R} t\right) \\
& +\frac{e}{2 \sin \theta_{W} \cos \theta_{W}} Z_{\mu}\left(e^{i \phi_{Z t c}^{L}} \kappa_{Z t c}^{L} \bar{c} \gamma^{\mu} P_{L} t\right. \\
& \left.+e^{i \phi_{Z t c}^{R} \kappa_{Z t c}^{R} \bar{c}} \gamma^{\mu} P_{R} t\right)+ \text { H.c. },
\end{aligned}
$$

which can be classified as two charged current operators which modify the SM top quark weak interactions with the $W$ boson and $b$ quark, as well as two flavor-changing neutral current (FCNC) operators involving the $Z$ boson, $t$, and $c$ quarks. Additional dimension $4 \mathrm{FCNC}$ operators with the $c$ quark replaced by the $u$ quark are also possible. We have included the $C P$ violating phases $\phi_{W t b(Z t c)}^{L(R)}$ in the interactions for generality, though they are not always considered in the literature.

We note that the anomalous $Z-t-c$ and $W-t-b$ interactions would have first appeared at dimension 6 if the electroweak symmetry were realized linearly. This would lead to the estimates $\kappa_{W t b}, \kappa_{Z t c} \sim \mathcal{O}\left(v^{2} / \Lambda^{2}\right)$ where $v$ is the electroweak symmetry breaking VEV. There are also operators such as $(\bar{t} c)(\bar{c} u)$, a four fermion contact interaction, which first appear at dimension 6 , the linearly realized situation should also include these possibilities in the analysis. These genuine dimension 6 operators have very different energy dependence than the dimension 4 operators we have considered, which might allow one to disentangle their contributions by careful study of the kinematics of the single top quark events.

In addition there are dimension 5 operators that involve interactions between new sets of particles and the top quark ${ }^{10}$ and can contribute to single top quark production. These include the FCNC operators

$$
\begin{aligned}
\mathcal{L}_{5}= & \frac{g_{S} G_{\mu \nu}^{a}}{\Lambda_{g t c}}\left(e^{i \phi_{g t c}^{L}} \kappa_{g t c}^{L} \bar{c} T^{a} \sigma^{\mu \nu} P_{L} t\right. \\
& \left.+e^{i \phi_{g t c}^{R} \kappa_{g t c}^{R} \bar{c}} T^{a} \sigma^{\mu \nu} P_{R} t\right) \\
& +\frac{2 e F_{\mu \nu}}{3 \Lambda_{\gamma t c}}\left(e^{i \phi_{\gamma t c}^{L} \kappa_{\gamma t c}^{L} \bar{c} \sigma^{\mu \nu} P_{L} t}\right. \\
& \left.+e^{i \phi_{\gamma t c}^{R} \kappa_{\gamma t c}^{R} \bar{c}} \sigma^{\mu \nu} P_{R} t\right)+ \text { H.c. },
\end{aligned}
$$

which couple the charm quark to the top and gluon or photon fields. Once again, we have included $C P$ violating phases $\phi_{g t c(\gamma t c)}^{L(R)}$ which are not generally considered in the literature. Additional operators with the charm replaced by the up quark are also possible. As dimension 5 operators, these terms have couplings with dimension of inverse mass that have been written in the form of $\kappa_{g t c}^{A} / \Lambda_{g t c}$ and $\kappa_{\gamma t c}^{A} / \Lambda_{\gamma t c}$, where $A=L, R$. In the discussion below, we will consider only the cases where all of these $\kappa$ couplings are 1 , and consider the magnitude allowed for the scales $\Lambda_{g t c}$ and $\Lambda_{\gamma t c}$. If the underlying theory is strongly coupled, these mass scales may be thought of as the energy scale in which the SM breaks down and must be replaced with the underlying theory. However, it should be kept in mind that if the underlying theory is weakly coupled, this interpretation is somewhat obscured by the fact that the energy scales $\Lambda$ will also include small factors of the fundamental (weak) interaction strength and loop suppression factors. Even in this case, an experimental constraint on $\Lambda$ is very useful because it will provide constraints on the parameters of an underlying model of new physics.

The dimension 4 terms which modify the $W-t-b$ vertex will clearly have a large impact on single top quark production [13]. However, $\kappa_{W t b}^{R}$ is already very strongly constrained by low energy $b \rightarrow s \gamma$ data [44], which requires [45]

$$
-0.0035 \geqslant\left(\kappa_{W t b}^{R} \cos \phi_{W t b}^{R}+20 \kappa_{W t b}^{R}{ }^{2}\right) \leqslant 0.0039,
$$

provided that $\kappa_{W t b}^{L}$ is smaller than 0.2. Further, the $C P$-violating observable that measures the asymmetry in the decay rates of $b \rightarrow s X$ and $\bar{b} \rightarrow \bar{s} X$ can test the imaginary part of the right-handed charged current coupling, i.e. $\kappa_{W t b}^{R} \sin \phi_{W t b}^{R}$ [45]. Given this strong constraint, it is unlikely

\footnotetext{
${ }^{10}$ There are also dimension five operators involving the sets of particles that already appear in Eq. (6) [42]. As discussed above, naive dimensional analysis suggests that these operators are less significant than their dimension four counterparts, so we limit $\mathcal{L}_{5}$ to the dimension 5 operators which involve only new sets of fields.
} 


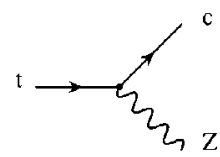

(a)

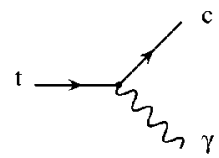

(b)

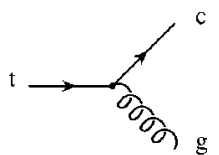

(c)
FIG. 14. Feynman diagrams showing FCNC top quark decays through (a) $t \rightarrow Z c$, (b) $t \rightarrow \gamma c$, and (c) $t \rightarrow g c$.

that further information about $\kappa_{W t b}^{R}$ can be gleaned from single top quark production, so we will assume $\kappa_{W t b}^{R}=0$ in the discussion below. Although the $b \rightarrow s \gamma$ process does not provide a good test of the left handed, $C P$-odd, $W-t-b$ coupling, i.e., $\kappa_{W t b}^{L} \sin \phi_{W t b}^{L}$, there are other $B$-decay processes with a good potential to measure it at future $B$ factories. In particular, the hadronic channels $B_{d} \longrightarrow \phi K_{s}$ and $B_{d} \rightarrow \Psi K_{s}$ have been considered in Ref. [46]. Assuming $\kappa_{W t b}^{R}=0$, all three single-top production modes are sensitive to $\kappa_{W t b}^{L}$, and will be proportional to $\left(1+\kappa_{W t b}^{L}{ }^{2} \cos ^{2} \phi_{W t b}^{L}+2 \kappa_{W t b}^{L} \cos \phi_{W t b}^{L}\right)$ much the same way that they will all be sensitive to $V_{t b}$ in the SM. ${ }^{11}$

The flavor-changing neutral current terms in $\mathcal{L}_{4}$ and $\mathcal{L}_{5}$ will also contribute to single top quark production, and since they involve particles lighter than the top quark mass, will also contribute to top quark decays through Feynman diagrams such as those shown in Fig. 14, which illustrate FCNC $t$ decays to $c$. The FCNC interactions between $t$ and $u$ will allow for exotic decays of the same type, but with the $c$ quark exchanged with a $u$ quark. One could hope to learn about these anomalous FCNC couplings both by studying single top quark production and top decays. However, this brings us back to the problem with using top quark decays to determine the magnitude of a coupling - the decay can provide information about the relative branching fraction of the exotic decay compared to the SM top quark decay $t$ $\rightarrow W^{+} b$, but since it does not allow one to measure the top quark decay width, it cannot provide a limit on the size of the exotic operator without first making an assumption concerning the nature of the $W-t-b$ interaction. In fact, one might think that the single top quark would suffer from the same difficulty in distinguishing the magnitude of new physics in the $W-t-b$ interaction from new physics in a FCNC interaction. However, as we shall see, one can use the three modes of single top quark production separately to disentangle the FCNC new physics from the possibility of $W-t-b$ new physics.

The three FCNC operators have a similar structure of a light $c$ (or $u$ ) quark interacting with a top quark and a neutral vector boson. Thus, we can discuss their impact on the three

\footnotetext{
${ }^{11}$ This is because the dimension 4 term that is proportional to $\kappa_{W t b}^{L}$ in $\mathcal{L}_{4}$ does not depend on the momenta of the interacting particles, as is the case for the SM $W-t-b$ interaction. For higher dimension $W-t-b$ operators, which may depend on the momenta, each single top quark mode will respond differently to the new interaction, and thus could be used to distinguish one operator from another.
}

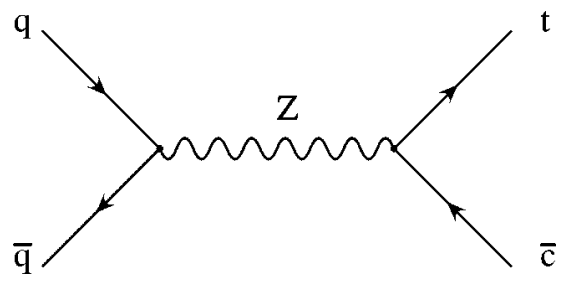

FIG. 15. Feynman diagram showing how a FCNC $Z-t-c$ interaction contributes to the $s$-channel mode of single top quark production through $q \bar{q} \rightarrow Z^{*} \rightarrow t \bar{c}$.

single top quark processes rather generally by considering the specific example of the $Z-t-c$ operator. In examining the FCNC operators in Eqs. (6) and (7), we note that they can have left-handed and right-handed interactions with different interaction coefficients (and even different phases). For now we will restrict our discussion to the case where all of the phases are zero, and discuss only the magnitude of the interactions, set by $\Lambda_{g t c}, \Lambda_{\gamma t c}$, and $\kappa_{Z t c}$. We will return to the subject of exploring their chiral structure when we consider top quark polarization in Sec. IV.

The $Z$ - $t$ - $c$ operator will allow for additional contributions to the $s$-channel mode of single top quark production through reactions such as $q \bar{q} \rightarrow Z^{*} \rightarrow t \bar{c}$, shown in Fig. 15 . This reaction has different initial and final state from the SM $s$-channel mode, and thus this new physics contribution does not interfere with the SM contribution to single top quark production. (The FCNC Z-t-c coupling can be induced at the loop level in the SM via the CKM mechanism, but its magnitude is small and can be neglected in our analysis.) The fact that the new physics process has a $\bar{c}$ instead of a $\bar{b}$ in the final state has a drastic practical consequence that the new physics production mechanism probably cannot be experimentally extracted at all, because in order to separate the $s$-channel mode from the large $t \bar{t}$ and $W$-gluon fusion backgrounds, it is necessary to tag the $\bar{b}$ produced in association with the top quark in the $s$-channel mode, in addition to the $b$ from the top quark decay. Thus, while a FCNC operator could contribute to $s$-channel production of a single top quark, it will not be counted as such. ${ }^{12}$

The $t W^{-}$mode cannot receive a contribution from a FCNC, though a FCNC will generally allow for new exotic production mechanisms such as $g c \rightarrow t Z$ shown in Fig. 16 [47]. From this consideration, along with the analysis of the $t W^{-}$mode in Sec. II, we see that the $t W^{-}$mode has a special quality because both the top and the $W$ are in the final state (and thus identifiable). Thus, it is sensitive to new phys-

\footnotetext{
${ }^{12}$ It could be possible to search for $s$-channel production via a FCNC with a specialized strategy differing from the usual one employed to extract the $W^{*}$ process, but such a search will require identifying the $\bar{c}$ produced in association with the top quark, and will suffer from large backgrounds from $t \bar{t}$ and $W$-gluon fusion single top quark processes.
} 

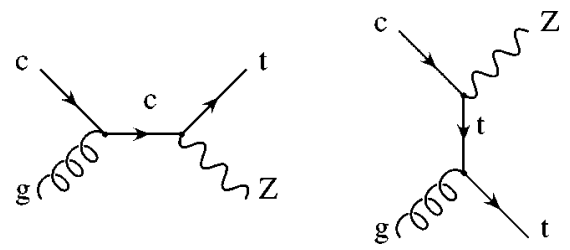

FIG. 16. Feynman diagrams showing how a FCNC $Z-t-c$ interaction contributes to the exotic mode of single top quark production $g c \rightarrow t Z$.

ics which modifies the $W-t-b$ interaction, ${ }^{13}$ but it is not sensitive to nonstandard physics involving new particles or FCNC's. Thus, the $t W^{-}$mode represents a chance to study the $W-t-b$ vertex without contamination from FCNC new physics.

The $W$-gluon fusion mode of single top quark production is quite sensitive to a FCNC involving the top quark and one of the light partons, through processes such as $c q \rightarrow t q$, from Feynman diagrams such as those shown in Fig. 17. The FCNC operators involve a different set of spectator quarks in the reaction, and thus they do not interfere with the SM $t$-channel process. In fact, because the $W$-gluon fusion mode requires finding a $b$ inside a hadron, which has less probability than finding a lighter parton, the FCNC's involving $u$ or $c$ quarks already receive an enhancement relative to the SM $t$-channel rate purely from the larger parton densities for the lighter flavors. This can somewhat compensate for a (presumably) smaller FCNC coupling. This shows the sense in which the $t$-channel single top quark mode is sensitive to the top quark's decay properties. The same type of new physics which opens up new top quark decay modes (and thus modifies the top quarks total width) will also modify the $t$-channel rate of single top quark production, because the same light partons into which the top quark may decay are also responsible for producing single top quarks in the $t$-channel process. Thus, one can think of the $t$-channel process as a kind of measure of the inclusive top quark width.

Because of the strong motivation to use single top quark production to study FCNC operators involving the top quark, detailed simulations of the effect of the $g-t-c$ operator on single top quark production were performed [48], and found that this operator could be constrained by the process $q \bar{q}$ $\rightarrow t \bar{c}$ to $\Lambda_{g t c} \geqslant 4.5 \mathrm{TeV}$ at Run II of the Tevatron if no new physics signal were to be found. Further refinements on this idea [49] showed that it could be improved by including other reactions such as $g c \rightarrow t, g c \rightarrow g t, q c \rightarrow t q$, and $g g$ $\rightarrow t \bar{c}$ to $\Lambda_{g t c} \geqslant 10.9 \mathrm{TeV}$ at the Tevatron Run II and to $\Lambda_{\text {gtc }} \geqslant 164 \mathrm{TeV}$ at the LHC.

Detailed simulations of the $Z-t-c$ and $\gamma-t-c$ operators have so far been confined to studies of top quark decays [50,52,51]. The left-handed Z-t-c FCNC operator, whose strength is parametrized in Eq. (6) by the quantity $\left|\kappa_{Z t c}^{L}\right|$ is constrained by low energy data on flavor-mixing processes

\footnotetext{
${ }^{13}$ Of course it is also sensitive to the $W-t-s$ and $W-t-d$ interactions.
}

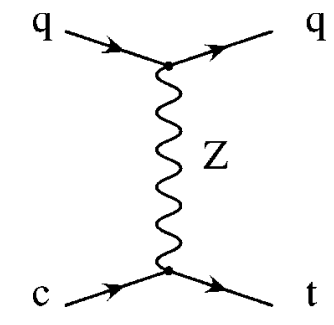

FIG. 17. Feynman diagram showing how a FCNC $Z-t-c$ interaction contributes to the $t$-channel mode of single top quark production through $c q \rightarrow t q$.

to be less than the order of magnitude of 0.05 [52]. The right-handed operator is more loosely constrained from low energy data by $\left|\kappa_{Z t c}^{R}\right| \leqslant 0.29$ [52]. While these limits are very interesting, they are indirect limits because all of the fields in the anomalous operators are virtual, and thus there could be cancellations between the $Z-t-c$ (or $\gamma-t-c$ ) operators and other nonstandard physics, and thus the single top quark production processes, as direct constraints, are independently valuable. Studies indicate that from Run II of the Tevatron, top quark decays should provide constraints of $\Lambda_{g t c} \geqslant 7.9$ $\mathrm{TeV}[50], \kappa_{Z t c}^{R} \leqslant 0.3$ [52], and will not improve the bounds on $\Lambda_{\gamma t c}$ from the current $b \rightarrow s \gamma$ limit of about $5 \mathrm{TeV}$ [51], unless a new physics signal is found. Of course, as we have argued before, it was necessary to assume a SM $W-t-b$ interaction in order to use decays to say anything at all about these operators. The effect of the $Z-t-c$ operator to the inclusive $t$-channel production rate is approximately independent of whether or not the operator is left-handed or right-handed, and thus in the discussion below we consider $\kappa_{Z t c}$, which can be taken either as $\kappa_{Z t c}^{L}$ or $\kappa_{Z t c}^{R}$. The effect of these operators on the $t$-channel cross section is to contribute an additional $0.13 \mathrm{pb}$ at the Tevatron Run II and $12.6 \mathrm{pb}$ at the LHC, assuming $\kappa_{Z t c}=0.29$, and including the NLO QCD corrections for both $t$ and $\bar{t}$ production. (These should be compared with the SM rates of $2.4 \mathrm{pb}$ and $243 \mathrm{pb}$, at Tevatron and LHC, respectively.)

As pointed out above, the constraints $\kappa_{Z t c}^{L}, \kappa_{Z t c}^{R}$ $<0.05,0.29$ were obtained by analyzing the low energy data with certain assumptions made for the underlying theory [52]. When additional new physics effect is added to the effect of the $\kappa_{Z t c}$ coupling to the low energy data, it may turn out that a large cancellation among various sources allow the size of $\kappa_{Z t c}$ to be at the order of 1 . Hence, a direct test at high energy colliders by studying the single top production is necessary to conclusively determine the coupling $\kappa_{Z t c}$. For $\kappa_{Z t c}=1$, the expected additional single top quark production rate for the $W$-gluon mode is $1.56 \mathrm{pb}$ at Run II of the Tevatron, and $146 \mathrm{pb}$ at the LHC, which in both cases would clearly be observable as deviations from the SM rates [17]. The $\gamma-t-c$ operator can be studied at a hadron collider through the reaction $\gamma c \rightarrow t$ (where the photon is treated as a parton inside the proton), though this exotic production mechanism suffers from potentially large SM backgrounds. In contrast, an electron Linear Collider will be better suited for this task. 


\section{POLARIZATION}

The polarization of top quarks represents another way to probe the properties of top quark interactions. In the SM, the $W-t-b$ vertex is entirely left-handed, which means that the top quark polarization information is passed on to the $W$ boson and $b$ quark into which the top quark decays. Since the $W$ interaction with the light fermions into which it decays is also left-handed, the $W$ polarization information is thus also reflected in the kinematics of its decay products. The same weak interaction is also responsible for single top quark production, which has the consequence that single top quarks also show a large degree of polarization. The discussion below is based on the SM amplitudes for top quark production and decay presented in [13].

\section{A. The $W^{+}$polarization: The $W-t-b$ interaction}

In order to probe the chiral structure of the $W-t-b$ interaction, it is enough to consider the $W$ polarization of top quark decays. As was shown in [13], the left-handed nature of the SM interaction demands that the produced $W$ bosons be either left-handed or longitudinally polarized, ${ }^{14}$ and predicts the fraction of the longitudinally polarized $W$ bosons from top quark decays to be

$$
f_{0}=\frac{m_{t}^{2}}{2 M_{W}^{2}+m_{t}^{2}} \simeq 70 \% .
$$

The degree of $W$ polarization from top quark decays can be reconstructed by studying the angle between the charged lepton momentum (defined in the $W$ rest frame) and the $W$ momentum defined in the top rest frame [13]. Since $t \bar{t}$ pairs are predominantly produced by QCD interactions $(q \bar{q}, g g \rightarrow t \bar{t})$, which conserve parity, the top quark is not polarized in its inclusive production, though there are correlations between the $t$ and $\bar{t}$ spin at the Tevatron because the dominant production is through a spin 1 gluon [43]. Hence, it is best to study top quark decays in $t \bar{t}$ events to test the SM lefthanded nature of the $W-t-b$ coupling by verifying the fraction of left-handed $W$ bosons from top quark decays to be $\left(1-f_{0}\right)$.

When probing the $W-t-b$ interaction from top quark decays, the $W$ and $b$ are observed, thus one can be sure that it is this interaction that is responsible for the effect one is seeing, which may not be the case if there is new physics in single top quark production. Once the chiral structure of the $W-t-b$ interaction is determined, one can then employ this information to unfold the top quark decay and reconstruct the polarization of the top quark itself, as will be explained below.

\section{B. The top quark polarization}

Once the chiral structure of the $W-t-b$ interaction has been probed through top quark decays, and the SM left-

\footnotetext{
${ }^{14} \mathrm{We}$ neglect the tiny mass of the bottom quark, as is justified given $\left(m_{b} / M_{W}\right)^{2} \sim 3.6 \times 10^{-4}$.
}

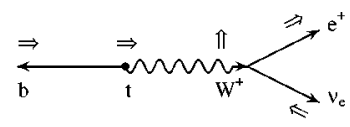

(a)

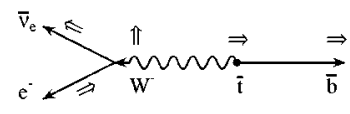

(c)

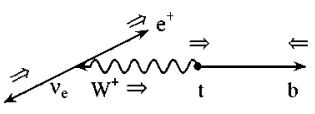

(b)

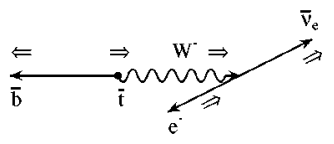

(d)
FIG. 18. A diagram indicating schematically the correlation between the charged lepton $\left(e^{ \pm}\right)$from a top quark decay, and the top quark spin, in the top quark rest frame. The arrows on the lines indicate the preferred direction of the momentum in the top quark rest frame, while the large arrows alongside the lines indicate the preferred direction of polarization. The figures correspond to top quark decay [(a) and (b)] and $\bar{t}$ decay [(c) and (d)] for the cases when the intermediate $W^{ \pm}$boson is longitudinally polarized [(a) and (c)] or left-handed [(b) and (d)]. In all cases, the $e^{+}\left(e^{-}\right)$from a $t(\bar{t})$ decay prefers to travel along (against) the direction of the $t$ $(\bar{t})$ polarization.

handed structure verified, the top quark decay products can be used in order to study the polarization of the produced top quarks themselves. As we will see, this can be very useful in determining what sort of new physics is responsible for an observed deviation in single top quark production. Currently, there are two important bases for describing the top quark polarization. The usual helicity basis measures the component of top quark spin along its axis of motion (in the center of mass frame-because the top quark mass is large, its helicity is not a Lorentz invariant quantity). The so-called "optimized basis" [54] relies on the SM dynamics responsible for single top quark production in order to find a direction (either along the direction of one of the incoming hadrons or produced jets) which results in a larger degree of polarization for the top quark. In the discussion below, we will describe the modes of single top quark production in both bases, and analyze the particular strengths and weaknesses of each.

Before looking at a particular process or basis, it is worth describing how one can determine the top quark polarization from its decay products in the decay mode of $t \rightarrow W^{+} b$ $\rightarrow l^{+} \nu_{l} b$ [13]. A simple heuristic argument based on the left-handed nature of the $W$ interactions and the conservation of angular momentum can be made, and is displayed diagrammatically in Fig. 18. The analysis is carried out in the rest frame of the top quark. When the $W$ boson is longitudinally polarized, it prefers to move in the same direction as the top spin, cf. Fig. 18(a). Its decay products prefer to align along the $W$ polarization, and since the $W$ is boosted in the direction of the top quark polarization, the charged lepton again prefers to move along the top quark spin axis. In the left-handed $W$ case, the fact that the $b$ quark must be lefthanded forces it to move along the direction of the top quark polarization, cf. Fig. 18(b). The $W$ thus moves against this direction. When the $W$ decays, the charged lepton $\left(l^{+}\right)$must be right-handed, so it prefers to move against the $W$ direction, in the same direction as the top quark polarization. Also shown in Figs. 18(c) and 18(d), a similar argument can be 
made for the $\bar{t}$ spin, but in this case the charged lepton prefers to move against the $\bar{t}$ spin axis. From this point onward, we restrict our discussion to top quarks, but it should be clear how they apply to $\bar{t}$ as well. The simple angular momentum argument is reflected in a more detailed computation of the distribution [53],

$$
\frac{1}{\Gamma} \frac{d \Gamma}{d \cos \theta}=\frac{1}{2}(1+\cos \theta),
$$

where $\theta$ is the angle between the direction of the charged lepton and the top quark polarization, in the top quark rest frame, and $\Gamma$ is the partial width for a semileptonic top quark decay in the SM. In principle, one has only to decide on a scheme for relating the top quark polarization to some axis, and one can fit the distribution,

$$
F(\cos \theta)=\frac{A}{2}(1+\cos \theta)+\frac{1-A}{2}(1-\cos \theta),
$$

to determine the degree of polarization $(A)$ along this axis. In practice, there are complications arising from the fact that the end points of the distribution tend to be distorted by the kinematic cuts required to isolate the signal from the background, and the fact that in reconstructing the top quark rest frame, the component of the unobserved neutrino momentum along the beam axis $\left(p_{\nu}^{z}\right)$ is unknown. One may determine this quantity up to a twofold ambiguity by requiring the top quark decay products to have an invariant mass that is close to $m_{t}$. However, the ambiguity in this procedure will also have some effect on the distribution, and so careful study is required. One can also use the asymmetry between events with $\cos \theta>0$ and $\cos \theta<0$ to characterize the degree of polarization of the top quark, which may be helpful if the data set is limited by poor statistics.

\section{1. $W^{*}$ production}

The degree of top quark polarization in the $s$-channel $W^{*}$ process is straightforward to compute in the helicity basis [13]. Using the CTEQ4M parton distribution functions (PDF's), we find that at the tree level about $75 \%$ of the top quarks produced through the $s$-channel process at the Tevatron are left-handed, and $76 \%$ of them are left-handed at the LHC [13].

The optimized basis improves the helicity basis result at the Tevatron by noting that in the SM, the $W^{*}$ process produces top quarks whose polarization is always along the direction of the initial antiquark involved in the scattering. At the Tevatron, the vast majority $(\sim 97 \%)$ of these antiquarks come from the $\bar{p}$ (which has valence antiquarks). Thus, one expects that by choosing to measure the top quark polarization along the $\bar{p}$ direction in the top quark rest frame, one can raise the degree of polarization from $75 \%$ to $97 \%$. This represents a large improvement for Tevatron polarization studies of the $W^{*}$ process. However, at the LHC there are no valence antiquarks, and thus no optimized basis to analyze the $W^{*}$ top polarization. In that case, the helicity basis is the sensible basis to analyze the polarization of the top quark, and results in a fair degree of left-handed top quark production.

\section{W-gluon fusion}

The discussion of polarization in the $W$-gluon fusion process is somewhat tricky, mostly owing to the fact that the detailed kinematics of this process are sensitive to higher orders of perturbation theory [12]. It is clear that the kinematic region described by the process $q b \rightarrow q^{\prime} t$ is the dominant one, but a precise calculation of the interplay between the $2 \rightarrow 2$ scattering contribution and the $2 \rightarrow 3$ scattering contribution is still lacking. Thus, one must be careful in claiming what degree of polarization results from a particular basis.

In the helicity basis, the $2 \rightarrow 2$ description has the top quarks $100 \%$ left-handed when produced from the $u b \rightarrow d t$ sub-process. In fact, at both Tevatron and LHC the $\bar{d} b$ $\rightarrow \bar{u} t$ subprocess is quite small, and thus the over-all degree of polarization is about $97 \%$. On the other hand, the $2 \rightarrow 3$ description shows a degree of polarization that is much lower, and depends on the choice of the regularization scheme for the collinear singularity (e.g., the bottom quark mass) used in the computation. This is an indication that this method of computation is not perturbatively stable. Thus, it is fair to say that the degree of polarization in the helicity basis is high, but at the moment no reliable determination is available. This situation should be improved by including higher order QCD corrections in $W$-gluon fusion simulation.

The optimized basis once again makes use of the fact that the top quark polarization is $100 \%$ along the direction of the spectator antiquark in the reaction. At both Tevatron and LHC, this is dominantly the spectator jet in the final state. This basis thus results in a top quark which is about $96 \%$ polarized along the direction of the spectator jet. In [54], it was shown that this basis is not sensitive to the value of the bottom quark mass, and thus is perturbatively reliable. In other words, higher order QCD effects are unlikely to have a large impact on the degree of top quark polarization in the optimized basis.

\section{New physics and top quark polarization}

As we have seen, new physics may alter the structure of single top quark production. It may be that the new physics effects will reveal themselves, and tell us something about their nature by causing a large deviation in one or more of the single top quark production cross sections. In that case one can study the distribution of the top quark polarization in order to learn something further about the nature of the nonstandard production mechanism.

In Sec. II, it was demonstrated that either a charged scalar top-quark pion or $W^{\prime}$ gauge boson can have a substantial effect on single top quark production in the $s$-channel mode. Assuming for the moment that such a deviation has been observed, one can then use the top quark polarization in order to narrow down the class of models responsible for such an effect. The $W^{\prime}$ boson couples to the left-handed top and bottom quarks, and thus an analysis of the resulting top 


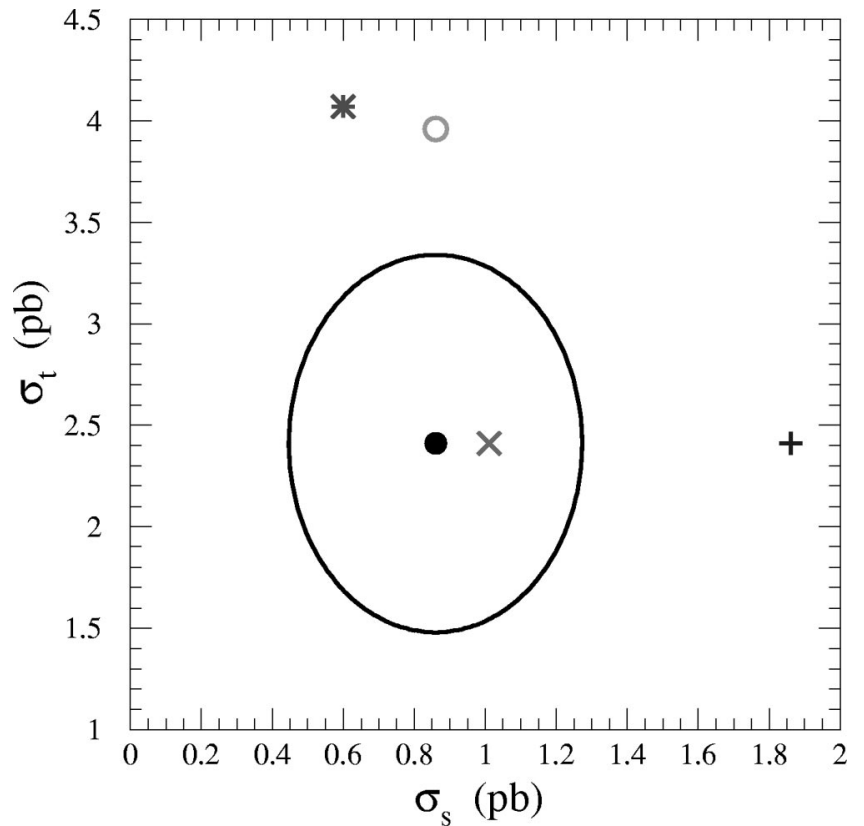

FIG. 19. The location of the Tevatron SM point (the solid circle) in the $\sigma_{s^{-}} \sigma_{t}$ plane, and the $3 \sigma$ theoretical deviation curve. Also shown are the points for the top-quark-flavor model (with $M_{Z}^{\prime}=1$ $\mathrm{TeV}$ and $\left.\sin ^{2} \phi=0.05\right)$ as the $\mathrm{X}$, the FCNC $Z-t-c$ vertex $\left(\left|\kappa_{Z t c}\right|=1\right)$ as the open circle, a model with a charged top-quark pion $\left(m_{\pi^{ \pm}}=250 \mathrm{GeV}\right.$ and $t_{R^{-}} c_{R}$ mixing of $\left.\sim 20 \%\right)$ as the cross, and a four quark generation scenario with $\left|V_{t s}\right|=0.55$ and $\left|V_{t b}\right|$ $=0.835$ as the asterisk. All cross sections sum the $t$ and $\bar{t}$ rates.

quark polarization will be similar to that of the SM prediction. Namely, the helicity basis will show about $75 \%$ of the tops to be left-handed ( $75 \%$ at the LHC), though the specific numbers show a mild sensitivity to the $W^{\prime}$ mass, and the optimized basis will show about $97 \%$ at the Tevatron. However, the $\pi^{ \pm}$has a right-handed interaction, completely at odds to the SM. In fact, there is another difference between the $W^{\prime}$ and the $\pi^{ \pm}$that is also very important. Like the SM $W$ boson, the $W^{\prime}$ is a vector particle, and thus carries angular momentum information between the initial state and final state in the $s$-channel process. However, the $\pi^{ \pm}$, as a scalar particle, does not carry such information. Thus, the optimized basis, which relies on the correlation between top spin and the initial $\bar{d}$ momentum fails to apply to a scalar production mechanism, and if one were to use it to analyze the polarization of the quark top coming from this type of new physics effect, one would come to the wrong conclusion that the produced tops were unpolarized. On the other hand, in the helicity basis the top quarks produced from the $\pi^{ \pm}$show very close to $100 \%$ right-handed polarization. This demonstrates the utility of using both bases. If there is new physics in single top quark production, not only is it unclear at the outset which basis will show a larger degree of polarization, but we can use them together to distinguish a vector from a scalar exchange, thus learning about the nature of the new particle without directly observing it.

Study of polarization can also be useful in disentangling the operators in the effective Lagrangian in Eqs. (6) and (7). As we saw, those operators have left-handed and right-

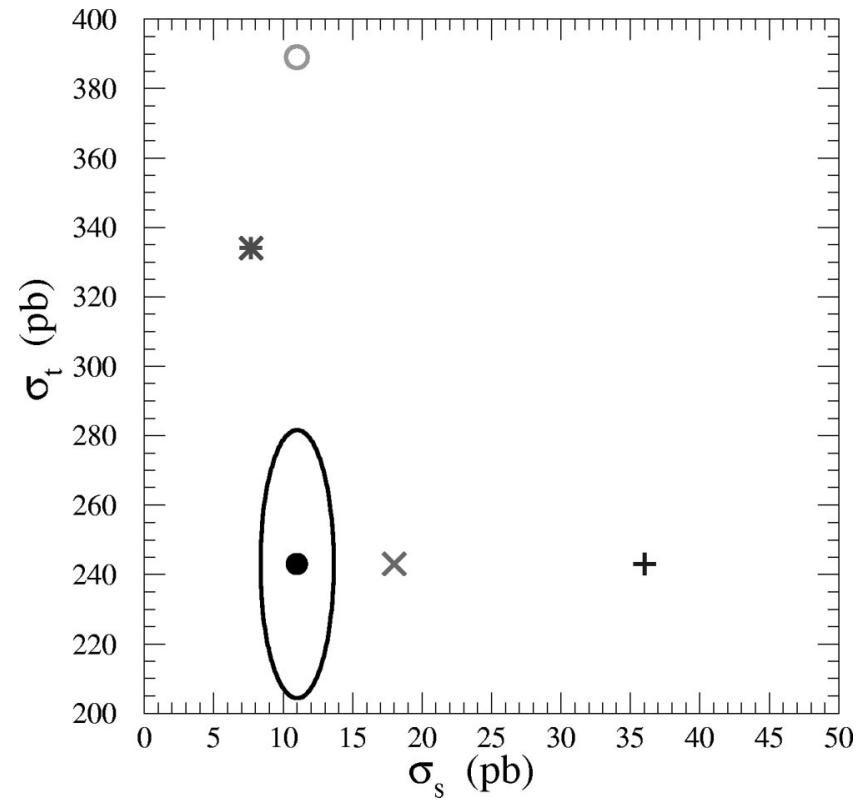

FIG. 20. The location of the CERN LHC SM point (the solid circle) in the $\sigma_{s}-\sigma_{t}$ plane, and the $3 \sigma$ theoretical deviation curve. Also shown are the points for the top-quark-flavor model (with $M_{Z}^{\prime}=1 \mathrm{TeV}$ and $\left.\sin ^{2} \phi=0.05\right)$ as the X, the FCNC $Z-t-c$ vertex $\left(\left|\kappa_{Z t c}\right|=1\right)$ as the open circle, a model with a charged top-quark pion ( $m_{\pi^{ \pm}}=450 \mathrm{GeV}$ and $t_{R^{-}} c_{R}$ mixing of $\left.\sim 20 \%\right)$ as the cross, and a four quark generation scenario with $\left|V_{t s}\right|=0.55$ and $\left|V_{t b}\right|$ $=0.835$ as the asterisk. All cross sections sum the $t$ and $\bar{t}$ rates.

handed versions, and thus the distribution of top quark polarizations will depend on the relative strength of the two. Thus, by studying top quark polarization, one could begin to disentangle the chiral structure of the operator responsible for a deviation in single top quark production, giving further insight into the nature of the full theory that accurately describes higher energies.

\section{CONCLUSIONS}

Having gone over in detail the physics one can probe with single top quark production, it is worth summarizing what we have learned and examining how one can use the different top quark observables to extract information about the top quark that maximizes the available information. In the preceding sections we have seen that single top quark production allows one to measure the magnitude of the top quark's weak interactions (in contrast to top quark decays). The three modes of single top quark production (the $s$-channel $W^{*}$, the $t$-channel $W$-gluon fusion, and the $t W^{-}$modes) are sensitive to different types of new physics. All three modes are sensitive to modification of the $W-t-b$ interaction, with the $t W^{-}$mode distinguished by the fact that it is rather insensitive to most types of new physics. The $s$-channel mode is sensitive to certain types of additional (heavy) particles. And the $t$-channel mode is sensitive to physics which modifies the top quark decay properties, in particular to FCNC interactions. In this light, it is rather unfortunate that the $t W^{-}$mode is so small at the Tevatron that it is not likely to be useful there, as it can allow one to 
measure the strength of the $W-t-b$ vertex, which would be a good first step in disentangling the information from the $s$ and $t$-channel modes.

Without the $t W^{-}$mode, one will most likely have to study the correlation of the $s$ - and $t$ - channel rates in the plane of $\sigma_{s}-\sigma_{t}$ in order to attempt to understand if a new physics effect is present, and how one should interpret it if it is observed. ( $\sigma_{s}$ and $\sigma_{t}$ are the cross sections of $W^{*}$ and $W$-gluon processes, respectively.) In Fig. 19 we show this plane for Run II of the Tevatron, including the SM point (with the contour of $3 \sigma$ theoretical uncertainty deviation around it) and the points from the top-quark-flavor model (with $M_{Z^{\prime}}=900 \mathrm{GeV}$ and $\sin ^{2} \phi=0.05$ ), the top-quark-color model with a charged top-quark pion (with mass $m_{\pi}^{ \pm}=250$ $\mathrm{GeV}$ and $t_{R}-c_{R}$ mixing of $20 \%$ ), a FCNC Z- $t$ - $c$ operator (with $\left|\kappa_{Z t c}\right|=1$ and $\phi_{Z t c}^{R}=\phi_{Z t c}^{L}=0$ ), and a large $V_{t s}$ under the four quark generation scenario with $\left|V_{t s}\right|=0.55$ and $\left|V_{t b}\right|=0.835$. A similar plot is given in Fig. 20 for the LHC. This illustrates how to use the knowledge we have about the sensitivity of the $W^{*}$ and $W$-gluon fusion modes to find a likely explanation for a new physics effect. A deviation in $\sigma_{s}$ that is not also reflected in $\sigma_{t}$ is most likely due to the effect of nonstandard particles. A deviation in $\sigma_{t}$ that is not also seen in $\sigma_{s}$ is likely from a FCNC. A deviation that is comparable in both rates is most likely from a modification of the $W-t-b$ interaction. In the very least, if the SM is a sufficient description of single top quark production, the fact that the two rates are consistent will allow one to use them to extract $V_{t b}$ with confidence that new physics is not distorting the measurement.

Additional information is provided by polarization information. By studying the $W$ polarization from top quark de- cays, one learns about the nature of the $W-t-b$ interaction. By studying the top quark polarization, in both the helicity and optimized bases, one can learn more about the chiral structure of nonstandard top quark interactions, either by probing the chiral structure of the interactions, or even the scalar/ vector nature of a virtual particle participating in single top quark production.

The large top quark mass seems to be a hint that the mechanism of the EWSB may be more evident in studies of the top quark than in other observables, and thus the Tevatron Run II and the LHC are exciting opportunities to probe the nature of the symmetry breaking. We have seen that by using the three modes of single top quark production together, along with studies of polarization in top quark decays and in single top quark production, one can assemble a coherent picture of the properties of the top quark. These observables are sensitive to different kinds of new physics, and thus when considered together can provide a probe of the nature of nonstandard physics manifest in the top quark sector, or can demonstrate the validity of the SM picture of how the top quark should behave.

\section{ACKNOWLEDGMENTS}

T.T. has benefitted from discussions with E.L. Berger, B.W. Harris, T. Lecompte, E. Malkawi, Z. Sullivan, and S. Willenbrock. We thank H.-J. He and W. Repko for collaboration on the associated production of a scalar boson with a single top quark. Work at Argonne National Lab is supported in part by the U.S. DOE under Contract No. W-31-109ENG-38. C.P.Y. is supported in part by the NSF under Grant No. PHY-9802564.
[1] CDF Collaboration, F. Abe et al., Phys. Rev. Lett. 74, 2626 (1995); D0 Collaboration, S. Abachi et al., ibid. 74, 2632 (1995).

[2] W.A. Bardeen, C.T. Hill, and M. Linder, Phys. Rev. D 41, 1647 (1990).

[3] C.T. Hill, Phys. Lett. B 345, 483 (1995); B.A. Dobrescu and C.T. Hill, Phys. Rev. Lett. 81, 2634 (1998); R.S. Chivukula, B.A. Dobrescu, H. Georgi, and C. T. Hill, Phys. Rev. D 59, 075003 (1999); M.B. Popovic and E.H. Simmons, ibid. 62, 035002 (2000); H.-C. Cheng, B.A. Dobrescu, and C.T. Hill, hep-ph/9912343; H. Collins, A. Grant, and H. Georgi, Phys. Rev. D 61, 055002 (2000); H. Georgi and A.K. Grant, ibid. 63, 015001 (2000) hep-ph/0006050; A. Aranda and C.D. Carone, Phys. Lett. B 488, 351 (2000).

[4] R.S. Chivukula, E.H. Simmons, and J. Terning, Phys. Lett. B 346, 284 (1995); E. Malkawi, Tim Tait, and C.-P. Yuan, ibid. 385, 304 (1996); D.J. Muller and S. Nandi, ibid. 383, 345 (1996); J.C. Lee, K.Y. Lee, and J.K. Kim, ibid. 424, 133 (1998); K.R. Lynch, E.H. Simmons, M. Narain, and S. Mrenna, hep-ph/0007286.

[5] E. Malkawi and C.-P. Yuan, Phys. Rev. D 61, 015007 (2000).

[6] H.-J. He, Tim Tait, and C.-P. Yuan, Phys. Rev. D 62, 011702 (2000).
[7] For a comprehensive introduction, Tim M.P. Tait, Ph.D. thesis, Michigan State University, hep-ph/9907462, 1999.

[8] M. Beneke et al., “Top quark physics,' hep-ph/0003033.

[9] C.A. Nelson and L.J. Adler, Jr., hep-ph/0006342; hep-ph/0007086.

[10] S. Mrenna and C.-P. Yuan, Phys. Rev. D 46, 1007 (1992).

[11] J. Bagger et al., "The case for a $500-\mathrm{GeV} e^{+} e^{-}$linear collider," hep-ex/0007022.

[12] S. Dawson, Nucl. Phys. B249, 42 (1985); S. Willenbrock and D. Dicus, Phys. Rev. D 34, 155 (1986); C.-P. Yuan, ibid. 41, 42 (1990); CCAST Symposium 1993, 259 (1993); Valencia Elem. Part. Phys. 1995, 148 (1995); “"5th Mexican Workshop of Particles and Fields," in Particles and Fields and Phenomenology of Fundamental Inteactions, Puebla, Mexico, 1995, edited by J. L. Lucio Martinez and M. Vargas, pp. 383-390; R.K. Ellis and S. Parke, Phys. Rev. D 46, 3785 (1992); G. Bordes and B. van Eijk, Z. Phys. C 57, 81 (1993); Nucl. Phys. B435, 23 (1995); T. Stelzer, Z. Sullivan, and S. Willenbrock, Phys. Rev. D 56, 5919 (1997).

[13] D.O. Carlson and C.-P. Yuan, Phys. Lett. B 306, 386 (1993); D.O. Carlson, Ph.D. thesis, Michigan State University, 1995.

[14] T. Stelzer, Z. Sullivan, and S. Willenbrock, Phys. Rev. D 58, 094021 (1998) 
[15] H. Lai, J. Huston, S. Kuhlmann, F. Olness, J. Owens, D. Sopher, W. K. Tung, and H. Weerts, Phys. Rev. D 55, 1280 (1997).

[16] S. Cortese and R. Petronzio, Phys. Lett. B 253, 494 (1991); T. Stelzer and S. Willenbrock, ibid. 357, 125 (1995); M.C. Smith and S. Willenbrock, Phys. Rev. D 54, 6696 (1996); S. Mrenna and C.-P. Yuan, Phys. Lett. B 416, 200 (1998).

[17] D.O. Carlson and C.-P. Yuan, Particle Phys. Phen. 1995, 172 (1995); Tim Tait and C.-P. Yuan, hep-ph/9710372.

[18] G. Ladinsky and C.-P. Yuan, Phys. Rev. D 43, 789 (1991); S. Moretti, ibid. 56, 7427 (1997); A.P. Heinson, A.S. Belyaev, and E.E. Boos, ibid. 56, 3114 (1997); A.S. Belyaev, E.E. Boos, and L.V. Dudko, ibid. 59, 075001 (1999); A.S. Belyaev and E. Boos, hep-ph/0003260.

[19] Tim M. P. Tait, Phys. Rev. D 61, 034001 (2000).

[20] D. Atwood, S. Bar-Shalom, G. Eilam, and A. Soni, hep-ph/0006032.

[21] For a review, S. Abel et al., "Report of the SUGRA working group for run II of the Tevatron,', hep-ph/0003154.

[22] C.-S. Li, R. Oakes, and J.-M. Yang, Phys. Rev. D 55, 1672 (1997); 55, 5780 (1997); C.-S. Li, R. Oakes, J.-M. Yang, and H.-Y. Zhou, ibid. 57, 2009 (1998); S. Bar-Shalom, D. Atwood, and A. Soni, ibid. 57, 1495 (1998).

[23] Particle Data Group, C. Caso et al., Eur. Phys. J. C 3, 1 (1998); 1999 off-year partial update for the 2000 edition available on the PDG WWW pages (URL: http://pdg.lbl.gov/).

[24] K. R. Dienes, E. Dudas, and T. Gherghetta, Phys. Lett. B 436, 55 (1998); Nucl. Phys. B537, 47 (1999).

[25] K. Agashe and M. Graesser, Phys. Rev. D 54, 4445 (1996).

[26] For a review, C. Kolda, Nucl. Phys. B (Proc. Suppl.) 62, 266 (1998).

[27] CDF Collaboration, J. Incandela, Fermilab-Conf-95/237-E (1995)

[28] G. Burdman, R.S. Chivukula, and N. Evans, Phys. Rev. D 62, 075007 (2000).

[29] E.H. Simmons, Phys. Rev. D 55, 5494 (1997).

[30] A. Datta, P.J. O’Donnell, Z.H. Lin, X. Zhang, and T. Huang, Phys. Lett. B 483, 203 (2000).

[31] H.-J. He and C.-P. Yuan, Phys. Rev. Lett. 83, 28 (1999).

[32] C. Balazs, H.-J. He, and C.-P. Yuan, Phys. Rev. D 60, 114001 (1999).

[33] G. Burdman, Phys. Rev. Lett. 83, 2888 (1999).

[34] P. Baringer, P. Jain, D.W. McKay, and L. Smith, Phys. Rev. D 56, 2914 (1997).

[35] R. Oakes, K. Whisnant, J.-M. Yang, B.-L. Young, and X. Zhang, Phys. Rev. D 57, 534 (1998).
[36] E.L. Berger, B.W. Harris, and Z. Sullivan, Phys. Rev. Lett. 83, 4472 (1999).

[37] J.L. Diaz-Cruz, M.A. Perez, and J.J. Toscano, Phys. Lett. B 398, 347 (1997).

[38] R. Peccei and X. Zhang, Nucl. Phys. B337, 269 (1990); E. Malkawi and C.-P. Yuan, Phys. Rev. D 50, 4462 (1994).

[39] A. Manohar and H. Georgi, Nucl. Phys. B234, 189 (1984).

[40] R. D. Peccei and X. Zhang, Nucl. Phys. B337, 269 (1990); R. D. Peccei, S. Peris, and X. Zhang, ibid. B349, 305 (1991).

[41] D. Carlson, E. Malkawi, and C.-P. Yuan, Phys. Lett. B 337, 145 (1994); E. Malkawi and C.-P. Yuan, Phys. Rev. D 50, 4462 (1994); 52, 472 (1995).

[42] F. Larios and C.-P. Yuan, Phys. Rev. D 55, 7218 (1997); F. Larios, Tim Tait, and C.-P. Yuan, ibid. 57, 3106 (1998); T. Han and J.L. Hewett, ibid. 60, 074015 (1999); E.R. Morales and M.E. Peskin, hep-ph/9909383; T. Han, Y.J. Kim, A. Likhoded, and G. Valencia, hep-ph/0005306.

[43] G. Mahlon and S. Parke, Phys. Rev. D 53, 4886 (1996); Phys. Lett. B 411, 173 (1997).

[44] T. Skwarnicki, talk at ICHEP98, Vancouver, Canada, 1998; CLEO Collaboration, M. Alam et al., Phys. Rev. Lett. 74, 2885 (1998).

[45] K. Chetyrkin, M. Misiak, and M. Munz, Phys. Lett. B 400, 206 (1997); F. Larios, M.A. Pérez, and C.-P. Yuan, ibid. 457, 334 (1999).

[46] A. Abd El-Hady and G. Valencia, Phys. Lett. B 414, 173 (1997).

[47] F. del Aguila, J.A. Aguilar-Saavedra, and L. Ametller, Phys. Lett. B 462, 310 (1999); F. del Aguila and J.A. AguilarSaavedra, Nucl. Phys. B576, 56 (2000).

[48] E. Malkawi and Tim Tait, Phys. Rev. D 54, 5758 (1996).

[49] M. Hosch, K. Whisnant, and B.-L. Young, Phys. Rev. D 56, 5725 (1997); T. Han, M. Hosch, K. Whisnant, B.-L. Young, and X. Zhang, ibid. 58, 073008 (1998).

[50] T. Han, K. Whisnant, B.-L. Young, and X. Zhang, Phys. Lett. B 385, 311 (1996); Tim Tait and C.-P. Yuan, Phys. Rev. D 55, 7300 (1997).

[51] T. Han, K. Whisnant, B.-L. Young, and X. Zhang, Phys. Rev. D 55, 7241 (1997).

[52] T. Han, R.D. Peccei, and X. Zhang, Nucl. Phys. B454, 527 (1995).

[53] M. Klein, H. Pietschmann, and H. Rupertsberger, Phys. Lett. 153B, 341 (1985); I. Bigi, Y. Dokshitzer, V. A. Khoze, J. Kühn, and P. Zerwas, Phys. Lett. B 181, 157 (1986); M. Jezabek and J. Kühn, ibid. 329, 317 (1994).

[54] G. Mahlon and S. Parke, Phys. Rev. D 55, 7249 (1997); Phys. Lett. B 476, 323 (2000). 